\title{
No Correlation between KRAS Status and Advanced Pancreatic Adenocarcinoma Survival
}

\author{
Misato Ogata ${ }^{1}$, Hironaga Satake ${ }^{1}$, Takatsugu Ogata ${ }^{1}$, Yukihiro Imai ${ }^{2}$, Yukimasa Hatachi ${ }^{1} \&$ Hisateru Yasui $^{1}$ \\ ${ }^{1}$ Department of Medical Oncology, Kobe City Medical Center General Hospital, 2-1-1, Minatojima \\ minamimachi, chuo-ku, Kobe city, Hyogo, 650-0047, Japan \\ ${ }^{2}$ Department of Pathology, Kobe City Medical Center General Hospital, 2-1-1, Minatojima minamimachi, \\ chuo-ku, Kobe city, Hyogo, 650-0047, Japan \\ Correspondence: Hironaga Satake, Department of Medical Oncology, Kobe City Medical Center General \\ Hospital, 2-1-1, Minatojima minamimachi, chuo-ku, Kobe city, Hyogo, 650-0047 Japan. E-mail: \\ takeh1977@gmail.com
}

Received: July 20, 2017

Accepted: August 5, $2017 \quad$ Online Published: October 13, 2017

doi:10.5539/cco.v6n2p45

URL: http://dx.doi.org/10.5539/cco.v6n2p45

\begin{abstract}
Erlotinib plus gemcitabine is one of the standard chemotherapies for unresectable pancreatic cancer. Pancreatic cancer has the highest frequency of KRAS gene mutations among human cancers, and some studies suggest that $K R A S$ status might be a predictive biomarker for anti-epidermal growth factor receptor treatment. However, the reliability of this biomarker has not been confirmed. Here, we evaluated the impact of KRAS mutations in pancreatic cancer patients treated with first line gemcitabine-based chemotherapy. 23 patients treated with gemcitabine-based chemotherapy whose KRAS status could be examined from primary or metastatic lesions were enrolled. KRAS mutations were analyzed by sequencing codons 12 and 13 . We retrospectively evaluated the correlation between KRAS status, and prognosis and treatment efficacy. Patient characteristics were as follows: median age 68 years, male/female=6/17, PS $0 / 1=9 / 14$, TNM stage III/IV=1/22, and gemcitabine alone/erlotinib plus gemcitabine=13/10. Among the 23 patients, KRAS codon 12 was mutated in 15 , one of whom also had mutation on codon 13. Median progression-free survival (PFS) and overall survival (OS) of all patients were 4.3 months (95\% confidence interval (CI): 3.1 to 5.4) and 8.1 months (95\% CI: 5.9 to 10.0; events in 96\%), respectively. KRAS status showed no association with PFS ( $\mathrm{p}=0.310)$, OS ( $\mathrm{p}=0.934)$, or the efficacy of treatment with $(\mathrm{p}=0.833)$ or without erlotinib $(\mathrm{p}=0.478)$. Thus, in this study, there was no correlation between KRAS status and the efficacy of first line chemotherapy with gemcitabine with or without erlotinib. Identification of a rationale for personalized medicine in pancreatic cancer will require further exploratory prospective studies.
\end{abstract}

Keywords: EGFR-TKI, erlotinib, gemcitabine, KRAS, pancreatic cancer

\section{Introduction}

Patients with pancreatic cancer have a 5-year survival rate of only 6\%. Approximately 330,000 people worldwide will die of pancreatic cancer annually, a number that is projected to double by 2030 (Torre et al., 2015).

The recommended treatment for advanced unresectable pancreatic adenocarcinoma is the FOLFIRINOX regimen or gemcitabine plus albumin-bound paclitaxel. The other option can be gemcitabine with erlotinib (GEM/erlotinib) (Ducreux et al., 2015).

The advantage of erlotinib is that it is an oral medicine and its side effects are less than those of cytotoxic agents. Erlotinib is an epidermal growth factor receptor tyrosine kinase inhibitor (EGFR-TKI), so if KRAS, which is located downstream of EGFR, has a mutation, it is reasonable to suggest that erlotinib has no effect. In fact, EGFR antibody does not have an effect on RAS mutation-type colorectal cancer. However, it is known that KRAS mutation status and the effectiveness of EGFR-TKI are not related in pancreatic cancer. Nevertheless, over $90 \%$ of pancreatic adenocarcinoma patients have KRAS mutations (Fitzgerald et al., 2015), so if KRAS mutation status could be a prognostic factor or a predictive factor for the efficacy of GEM/erlotinib, we could select better treatment choices according to the KRAS mutation status. Since it remains unclear if KRAS mutation status is associated with the effect of erlotinib, we decided to evaluate it. 


\section{Materials and methods}

\subsection{Patients and Data Collections}

We retrospectively studied 23 consecutive patients in a single center, Kobe City Medical Center General Hospital, with advanced unresectable pancreatic adenocarcinoma from January 2011 to December 2012. They were treated with GEM/erlotinib or GEM as first-line treatment, and we could identify if they were either KRAS mutation type or wild-type. KRAS mutation was analyzed by PCR-rSSO method. GEM/erlotinib consists of gemcitabine $1,000 \mathrm{mg} / \mathrm{m}^{2}$ on days $1,8,15$ and erlotinib $100 \mathrm{mg}$ on days $1-28$ monthly, and GEM consists of gemcitabine $1,000 \mathrm{mg} / \mathrm{m}^{2}$ on days $1,8,15$ monthly. The response evaluation criteria for this study are based on response evaluation criteria in solid tumor (RECIST) 1.1, and the side effect is based on national cancer institute - common toxicity criteria (NCI-CTC) ver. 4.0. Overall response rate (ORR) means complete response (CR) plus partial response (PR) and disease control rate (DCR) means CR plus PR plus stable disease (SD). The progression-free survival (PFS) was defined as the time from the date of the first administration of chemotherapy to the first documentation of disease progression, subsequent therapy, or death. Overall survival (OS) was determined from the date of the first administration of chemotherapy to the date of death or last confirmation of survival.

\subsection{Statistics}

Statistical analysis of the long-term outcomes was done with the Kaplan-Meier method. Continuous data were compared with the Mann-Whitney U test. The Pearson chi-squared test or Fisher's exact test was used to analyze categorical data and compare proportions.

Statistical data were obtained using the SPSS software package (SPSS 22.0 Inc., Chicago, IL). Statistical significance was defined as a $P$ value of less than 0.05 .

\section{Results}

\subsection{Patient Characteristics}

The characteristics of the patients are listed in Table 1. Fifteen patients (65\%) had KRAS mutations and one patient had both KRAS exon 2 codon 12 and codon 13 mutations.

Table 1. Patient characteristics $(\mathrm{n}=23)$

\begin{tabular}{lll}
\hline Age $($ year) & median (range) & $68(49-83)$ \\
\hline Sex & male/female & $6 / 17$ \\
ECOG PS & $0 / 1$ & $9 / 14$ \\
cStage UICC & III/IV & $1 / 22$ \\
Metastasis & $0 / 1 />1$ & $9 / 11 / 3$ \\
Peritoneal dissemination & Yes & 8 \\
KRAS codon12/13 & WT/12MT/13MT & $8 / 15 / 1$ \\
First line chemotherapy & GEM/GEM+erlotinib & $13 / 10$ \\
CA19-9, U/ml & median (range) & $348.2(2-87300)$ \\
Primary site of disease & head/body/tail/unknown & $7 / 8 / 7 / 1$ \\
Tumor grade & well/moderately/poor/unknown & $5 / 6 / 3 / 9$ \\
\hline
\end{tabular}

Note. ECOG, Eastern Cooperative Oncology Group; PS, performance status; UICC, Union for International Cancer Control; CA19-9, carbohydrate antigen 19-9; WT, wild-type; MT, mutation type; GEM, gemcitabine; GEM+erlotinib, gemcitabine plus erlotinib

\subsection{KRAS Wild-type Versus KRAS Mutation Type}

Patient characteristics classified by KRAS status are listed in Table 2. The characteristics were well-balanced between the two arms, so we can safely say that we need no adjustment. 
Table 2. Patient characteristics classified by KRAS status

\begin{tabular}{lllll}
\hline & & KRAS WT $(\mathrm{n}=8)$ & KRAS MT $(\mathrm{n}=15)$ & $\mathrm{p}$-value \\
\hline Age(year) & median (range) & $69.5(57-83)$ & $65(49-76)$ & .400 \\
Sex & male/female & $3 / 5$ & $3 / 12$ & .506 \\
ECOG PS & $0 / 1$ & $5 / 3$ & $4 / 11$ & .169 \\
cStage UICC & III/IV & $0 / 8$ & $1 / 14$ & .825 \\
Metastasis & $0 / 1 />1$ & $4 / 3 / 1$ & $5 / 8 / 2$ & .591 \\
$1^{\text {st }}$ line chemotherapy & GEM/GEM+erlotinib & $5 / 3$ & $6 / 9$ & .728 \\
CA19-9, U/ml & median (range) & $280.4(2-62200)$ & $777(8-87300)$ & .667 \\
Primary site of disease & head/body/tail/unknown & $3 / 1 / 4$ & $4 / 7 / 3 / 1$ & .825 \\
Tumor grade & well/moderately/poor/unknown & $1 / 2 / 2 / 3$ & $4 / 4 / 1 / 6$ & .681 \\
\hline
\end{tabular}

Note. ECOG, Eastern Cooperative Oncology Group; PS, performance status; UICC, Union for International Cancer Control; CA19-9, carbohydrate antigen 19-9; WT, wild type; MT, mutation type; GEM, gemcitabine; GEM+erlotinib, gemcitabine plus erlotinib; WT, wild-type; MT, mutation type

\subsection{Using Erlotinib or not}

Patient characteristics classified by erlotinib use are listed in Table 3 .

Table 3. Patient characteristics classified by erlotinib use

\begin{tabular}{lllll}
\hline & & $\begin{array}{l}\text { With erlotinib } \\
(\mathrm{n}=10)\end{array}$ & $\begin{array}{l}\text { Without } \\
\text { erlotinib (n=13) }\end{array}$ & p-value \\
\hline Age(year) & median (range) & $69(53-74)$ & $65(49-83)$ & .999 \\
Sex & male/female & $2 / 8$ & $4 / 9$ & .693 \\
ECOG PS & $0 / 1$ & $2 / 8$ & $7 / 6$ & .186 \\
cStage UICC & III/IV & $1 / 9$ & $0 / 13$ & .693 \\
Metastasis & $0 / 1 />1$ & $4 / 5 / 1$ & $5 / 6 / 2$ & .879 \\
Peritoneal dissemination & Yes & 3 & 5 & .738 \\
KRAS & WT/codon12MT/13MT & $3 / 7 / 1$ & $5 / 8 / 0$ & .728 \\
CA19-9, U/ml & median (range) & $236.9(2-62200)$ & $1412(8-87300)$ & .214 \\
Primary site of disease & head/body/tail/unknown & $3 / 5 / 2 / 0$ & $4 / 3 / 5 / 1$ & .446 \\
Tumor grade & well/moderately/poor/unknown & $1 / 3 / 1 / 5$ & $4 / 3 / 2 / 4$ & .313 \\
\hline
\end{tabular}

Note. ECOG, Eastern Cooperative Oncology Group; PS, performance status; UICC, Union for International Cancer Control; CA19-9, carbohydrate antigen 19-9; WT, wild-type; MT, mutation type; WT, wild type; MT, mutation type

\subsection{Side Effects}

Table 4. Side effects which showed a difference between the two arms

\begin{tabular}{llllll}
\hline & \multicolumn{4}{l}{ With erlotinib $(\mathrm{n}=10)$} & \multicolumn{3}{l}{ Without erlotinib $(\mathrm{n}=13)$} & \\
\hline & ALL, $\%$ & $3 / 4, \%$ & ALL, $\%$ & $3 / 4, \%$ & p-value \\
Acne-like rash & 60 & 0 & 15 & 0 & .077 \\
Diarrhea & 60 & 0 & 15 & 0 & .077 \\
Interstitial pneumonia & 20 & 0 & 0 & 0 & .446 \\
Gastrointestinal hemorrhage & 10 & 10 & 0 & 0 & .693 \\
Gastrointestinal perforation & 10 & 10 & 0 & 0 & .693 \\
\hline
\end{tabular}

The worst toxicities related to the chemotherapy are listed in Table 4. The hematological side effects did not differ significantly between the two arms of KRAS mutation and KRAS wild-type.

\subsection{Clinical Outcomes}

The response rates are summarized in Table 5 .

The median PFS of all patients was 4.3 months and the median OS was 8.1 months. With regard to KRAS status, the median PFS of KRAS wild-type patients was 3.6 months, and that of KRAS mutation type patients was 3.9 months ( $\mathrm{p}=0.310$ ). The median OS of KRAS wild-type patients was 6.0 months, and that of KRAS mutation type patients was 8.6 months ( $\mathrm{p}=0.934$ ). The median PFS of those treated with erlotinib was 3.6 months, and that of 
those treated without erlotinib was 5.1 months $(\mathrm{p}=0.360)$. The median OS of those treated with erlotinib was 8.6 months, and that of those treated without erlotinib was 7.1 months $(\mathrm{p}=0.833)$. Regarding the patients treated without erlotinib, the PFS of KRAS wild-type and mutation type patients were 2.0 months and 6.0 months, respectively ( $\mathrm{p}=0.566$ ), and the OS of KRAS wild-type and mutation type patients were 7.0 months and 8.1 months, respectively ( $\mathrm{p}=0.478$ ). Regarding the patients treated with erlotinib, the PFS of KRAS wild-type and mutation type patients were 3.6 months and 4.3 months, respectively ( $\mathrm{p}=0.774$ ), and the OS of KRAS wild-type and mutation type patients were 6.0 months and 9.8 months, respectively $(\mathrm{p}=0.084)$.

Table 5. Response rate

\begin{tabular}{|c|c|c|c|c|c|}
\hline & \multicolumn{2}{|l|}{$\mathrm{n}$} & \multicolumn{3}{|l|}{$\%$} \\
\hline CR & \multicolumn{2}{|l|}{0} & \multicolumn{3}{|l|}{0} \\
\hline PR & \multicolumn{2}{|l|}{4} & \multicolumn{3}{|c|}{17} \\
\hline SD & \multicolumn{2}{|l|}{12} & \multicolumn{3}{|c|}{52} \\
\hline PD & \multicolumn{2}{|l|}{7} & \multicolumn{3}{|c|}{30} \\
\hline ORR (CR+PR) & \multicolumn{2}{|l|}{4} & \multicolumn{3}{|c|}{17} \\
\hline \multirow[t]{3}{*}{$\mathrm{DCR}(\mathrm{CR}+\mathrm{PR}+\mathrm{SD})$} & \multicolumn{2}{|l|}{16} & \multicolumn{3}{|c|}{70} \\
\hline & \multicolumn{2}{|c|}{ With erlotinib $(n=10)$} & \multicolumn{3}{|c|}{ Without erlotinib $(n=13)$} \\
\hline & $\mathrm{n}$ & $\%$ & $\mathrm{n}$ & $\%$ & p-value \\
\hline CR & 0 & 0 & 0 & 0 & .999 \\
\hline PR & 2 & 20 & 2 & 15 & .879 \\
\hline SD & 6 & 60 & 6 & 46 & 605 \\
\hline PD & 2 & 20 & 5 & 38 & 483 \\
\hline ORR (CR+PR) & 2 & 20 & 2 & 15 & .879 \\
\hline $\mathrm{DCR}(\mathrm{CR}+\mathrm{PR}+\mathrm{SD})$ & 8 & 80 & 8 & 62 & .483 \\
\hline
\end{tabular}

Note. CR: complete response, PR: partial response, SD: stable disease, PD: progressive disease, ORR: overall response rate, DCR: disease control rate

\section{Discussion}

It is well known that gemcitabine plus albumin-bound paclitaxel, the standard treatment for advanced unresectable pancreatic adenocarcinoma, has a side effect such as numbness, which has a large influence on patients' quality of life. If we could learn whether erlotinib were likely to produce a better outcome for a patient only by searching the KRAS mutation status, it would be to the patient's benefit. Therefore, it would be an advantage if a predictive factor for the effect of erlotinib-combined chemotherapy could be established. According to the NCCN Guidelines Version 1. 2017, GEM/erlotinib is recommended for locally advanced or unresectable pancreatic cancer in patients with good performance status, although only a small subset of patients benefit. Also, erlotinib is used for non-small-cell lung cancer with EGFR mutation, and pre-clinical evidence suggested that EGFR intron 1 polymorphism might mediate the erlotinib response (Tzeng et al., 2007), so we can possibly find that pancreatic cancer patients with EGFR mutation also have a good response by using erlotinib.

In this study, we did not find a relationship between KRAS mutation status and the effectiveness of erlotinib. One reason might be that pancreatic cancer patients have many mutations (Jones et al., 2008), and a KRAS mutation may not be the only carcinogenic signal, so an EGFR-TKI is actually not effective. Moreover, a prospective, randomized, double-blind, multicenter phase II trial on the effectiveness of vandetanib in locally advanced or metastatic pancreatic carcinoma was published in March, 2017 (Middleton et al., 2017). This trial suggests that the addition of vandetanib to gemcitabine monotherapy does not improve the OS in advanced pancreatic cancer. Vandetanib is a tyrosine kinase inhibitor of vascular endothelial growth factor receptor 2, RET, and EGFR, so this result suggests that it may be difficult for erlotinib, which only inhibits EGFR, to show a significant improvement. Although EGFR-TKI and EGFR antibody both inhibit EGFR signaling, their mechanisms differ. EGFR antibodies bind to the extracellular domain of the EGFR and inhibit binding of other growth factor ligands. Another important mechanism is that EGFR disappears from the cell surface in a process called antibody-receptor internalization. This completely prevents ligand-induced autophosphorylation of the EGFR. On the other hand, TKIs bind to the catalytic domain of the EGFR within the cell to block the downstream effect and, moreover, inhibition by erlotinib is reversible. Taking these differences into consideration, it is no wonder that erlotinib does not work like an EGFR antibody, which is not effective against $R A S$ mutation-type colorectal cancer. 
However, a retrospective study in Korea showed a worse outcomes of GEM/erlotinib combination chemotherapy in patients with KRAS mutations compared with patients with wild-type KRAS, which is comparable with the molecular analysis of the trial, AIO-PK0104 (Kim et al., 2011). This trial is a randomized cross-over phase III trial, which showed that KRAS wild-type patients treated with erlotinib combined with either gemcitabine or capecitabine had an improved OS over that of KRAS mutant patients. Post hoc subgroup analysis of the AIO-PK0104 showed that a trend for a survival benefit was also observed during subsequent chemotherapy (Boeck et al., 2013). Furthermore, Shao et al. (2014) reported the expression of KRAS mutations in vitro showing no effects on erlotinib, while resulting in a reduced sensitivity to gefitinib, another well-known inhibitor of the tyrosine kinase domain of EGFR. These results support the assumption that KRAS status is rather a prognostic than a predictive in advanced pancreatic cancer, and Hayashi et al. (2017) reported the number of major driver genes mutations in pancreatic cancer (KRAS, CDKN2, TP53 and CDKN2A) might be a promising prognostic biomarker.

Recently, refametinib, an orally available selective inhibitor of MEK1/2, has shown a promising objective response rate in $K R A S$ wild-type patients in combination with GEM (Van Laethem et al., 2017). This study may influence the treatment decision of KRAS wild-type patients in the future.

Other gene mutations such as BRCA2 (6-12\%), PALB2 (1-3\%), BRCA1, CDKN2A, STK11, PRSS1, SPINK1, MSH2, FANC-C are known to occur in pancreatic cancer patients. For example, in the laboratory, pancreatic cancer cell lines with mutations in the BRCA2 gene are sensitive to poly ADP-ribose polymerase inhibitors, resulting in the death of cancer cells (Hruban, Canto, Goggins, Schulick, \& Klein, 2010). This is now being translated to the clinic: Olaparib, which is the first poly ADP-ribose polymerase inhibitor to be approved as maintenance monotherapy for the treatment of patients with platinum-sensitive relapsed BRCA-mutated serous ovarian cancer. Similarly, if we could find an agent that leads KRAS mutated cells to death, and conduct a clinical trial, it could be a better choice for KRAS mutant patients.

Our limitations of this study are a small sample size and that it is conducted at single center retrospectively. Also, in this study, patient characteristics of KRAS mutant arm tend to be younger and tumor grade of KRAS mutant arm tends to be better, which may lead to somewhat longer PFS and OS in KRAS mutant patients.

\section{Conclusion}

In this study, there was no correlation between KRAS status and the efficacy of $1^{\text {st }}$ line chemotherapy with gemcitabine with or without erlotinib. Identification of a rationale for personalized medicine in pancreatic cancer will require further exploratory prospective studies.

\section{Acknowledgments}

Written informed consent was obtained from the patients for publication of this paper. The authors would like to thank the patients and families who participated in this study.

\section{References}

Boeck, S., Jung, A., Laubender, R. P., Neumann, J., Egg, R., Goritschan, C., ... Heinemann, V. (2013). KRAS mutation status is not predictive for objective response to anti-EGFR treatment with erlotinib in patients with advanced pancreatic cancer. J Gastroenterol, 48(4), 544-548. https://doi.org/10.1007/s00535-013-0767-4

Ducreux, M., Cuhna, A. S., Caramella, C., Hollebecque, A., Burtin, P., Goere, D., ... Arnold, D. (2015). Cancer of the pancreas: ESMO Clinical Practice Guidelines for diagnosis, treatment and follow-up. Ann Oncol, 26 Suppl 5, v56-68. https://doi.org/10.1093/annonc/mdv295

Fitzgerald, T. L., Lertpiriyapong, K., Cocco, L., Marteli, A. M., Libra, M., Candido, S., ... McCubrey, J.A. (2015). Roles of EGFR and KRAS and their downstream signaling pathways in pancreatic cancer and pancreatic cancer stem cells. Adv Biol Regul, 59, 65-81. https://doi.org/10.1016/j.jbior.2015.06.003

Hayashi, H., Kohno, T., Ueno, H., Hiraoka, N., Kondo, S., Saito, M., ... Okusaka, T. (2017). Utility of Assessing the Number of Mutated KRAS, CDKN2A, TP53, and SMAD4 Genes Using a Targeted Deep Sequencing Assay as a Prognostic Biomarker for Pancreatic Cancer. Pancreas, 46(3), 335-340. https://doi.org/10.1097/MPA.0000000000000760

Hruban, R. H., Canto, M. I., Goggins, M., Schulick, R., \& Klein, A. P. (2010). Update on familial pancreatic cancer. Adv Surg, 44, 293-311.

Jones, S., Zhang, X., Parsons, D. W., Lin, J. C., Leary, R. J., Angenendt, P., ... Kinzler, K. W. (2008). Core signaling pathways in human pancreatic cancers revealed by global genomic analyses. Science, 321, 
1801-1806. https://doi.org/10.1126/science.1164368

Kim, S. T., Lim, D. H., Jang, K. T., Lim, T., Lee, J., Choi, Y. L., .. Park, J. O. (2011). Impact of KRAS mutations on clinical outcomes in pancreatic cancer patients treated with first-line gemcitabine-based chemotherapy. Mol Cancer Ther, 10, 1993-1999. https://doi.org/10.1158/1535-7163.MCT-11-0269

Middleton, G., Palmer, D. H., Greenhalf, W., Ghaneh, P., Jackson, R., Cox, T., ... Neoptolemos, J. P. (2017). Vandetanib plus gemcitabine versus placebo plus gemcitabine in locally advanced or metastatic pancreatic carcinoma (ViP): a prospective, randomised, double-blind, multicentre phase 2 trial. Lancet Oncol, 18, 486-499. http://dx.doi.org/10.1016/S1470-2045(17)30084-0

Shao, T., Zheng, Y., Zhao, B., Li, T., Cheng, K., \& Cai, W. (2014). Recombinant expression of different mutant K-ras gene in pancreatic cancer Bxpc-3 cells and its effects on chemotherapy sensitivity. Sci. China Life Sci, 57, 1011-1017. https://doi.org/10.1007/s11427-014-4724-0

Torre, L. A., Bray, F., Siegel, R. L., Ferlay, J., Lortet-Tieulent, J., \& Jemal, A. (2015). Global cancer statistics, 2012. CA: A Cancer Journal for Clinicians, 65, 87-108. http://dx.doi.org/10.3322/caac.21262

Tzeng, C. W., Frolov, A., Frolova, N., Jhala, N. C., Howard, J. H., Vickers, S. M., \& Arnoletti, J. P. (2007). Pancreatic cancer epidermal growth factor receptor (EGFR) intron 1 polymorphism influences postoperative patient survival and in vitro erlotinib response. Ann Surg Oncol, 14, 2150-2158. http://doi.org/10.1245/s10434-007-9409-5

Van Laethem, J. L., Riess, H., Jassem, J., Haas, M., Martens, U. M., Weekes, C., ... Childs, B. H. (2017). Phase I/II Study of Refametinib (BAY 86-9766) in Combination with Gemcitabine in Advanced Pancreatic cancer. Target Oncol, 12, 97-109. http://doi.org/10.1007/s11523-016-0469-y

\section{Copyrights}

Copyright for this article is retained by the author(s), with first publication rights granted to the journal.

This is an open-access article distributed under the terms and conditions of the Creative Commons Attribution license (http://creativecommons.org/licenses/by/4.0/). 